\title{
Altered neurogenesis and disrupted expression of synaptic proteins in prefrontal cortex of SHANK3-deficient non-human primate
}

\author{
Cell Research (2017) 27:1293-1297. doi:10.1038/cr.2017.95; published online 25 July 2017
}

\section{Dear Editor,}

Despite substantial progress made toward understanding the molecular changes contributing to autism spectrum disorders (ASD), the neuropathophysiology underlying ASD remains poorly understood $[1,2]$. Structural brain imaging in humans is valuable, but lacks resolution at the cellular level. Studies of neuropathology in humans have been hampered by the lack of high quality postmortem brains from individuals with ASD [2]. For more than decades, mutant mice have served as major tools to dissect the pathophysiology of ASD because of the wealth of molecular and neurobiological techniques developed for studies with rodents. Our knowledge of molecular and cellular mechanisms for ASD is mostly limited to what we have learned from genetically modified mice. However, there are significant evolutionary differences in brain structure and behavior between rodents and humans. For example, social behaviors and the organization of cerebral cortex differ significantly between primates and rodents. The cerebral neocortex comprises $\sim 80 \%$ of the human brain and $\sim 72 \%$ of the macaque brain, but only $\sim 28 \%$ of the rat brain. Prefrontal cortex (PFC), a critical region for high order cognitive and social functions, is under-developed in rodents compared with primates. The unique behavioral features in human ASD have posed significant challenges to assess the translational value of many findings from ASD mouse models. The apparent evolutionary differences in brain structures and behaviors between mouse and human highlight the need of alternative animal models such as non-human primate models for ASD [3]. The value of a primate model to study ASD has been supported by the generation and characterization of monkeys with altered expressions of $M E C P 2$ using the lentivirus- and TALENbased method $[4,5]$.

Mutations in human SHANK3 remain one of the best-replicated and well-characterized genetic defects in ASD. Various types of mutations in SHANK3 have been found in $\sim 1 \%$ of individuals with ASD [6]. SHANK3 protein is one of the three-member family proteins (SHANK1-3) known to function as postsynaptic scaffolding proteins in excitatory synapses. So far, a total of 14 independent lines of Shank3-mutant mice have been reported, but none of these studies focused on the neuropathology in the prenatal brain $[6,7]$. These mutant mice display a spectrum of behavioral phenotypes. However, interpretation of the behavioral phenotypes in rodent models relevant to the SHANK3-related disorders remains a challenge. For these reasons, SHANK3 represented a unique opportunity to test the feasibility of generating an ASD model in non-human primates.

We used the Cas9/sgRNA method to disrupt SHANK3 gene in cynomolgus monkeys (Macaca fascicularis) following previous protocols $[8,9]$. SHANK3 has an array of mRNA isoforms due to multiple promoters and extensive alternative splicing of coding exons in both human and rodent, and this pattern is expected to be conserved in non-human primates $[5,10]$. We therefore simultaneously targeted two sites (exons 6 and 12) of SHANK3 to induce a large deletion (Figure 1A and Supplementary information, Figure S1A) that could completely disrupt all SHANK3 isoforms. We selected sgRNAs that showed high targeting efficiency for injection into monkey embryos.

We obtained 3 pregnancies after transferring 116 injected embryos to 37 surrogate mothers (Figure 1B). The pregnancy rate of $8.1 \%(3 / 37)$ was significantly lower than that of other targeted genes $(34.5 \%)$ performed in the same center under the same experimental conditions (Li X et al., unpublished data). The reason for the low pregnancy rate is currently unknown, but suggests that SHANK3 expression might be important for early development in primates. Of three monkey offsprings, one miscarried close to the term gestation (125 days) $\left(S H A N K 3^{M I}\right)$, one was lost due to a difficult labor $\left(S H A N K 3^{M 2}\right)$, and one is still alive $\left(S H A N K 3^{M 3}\right)$ (Supplementary information, Figure S1B). There are 

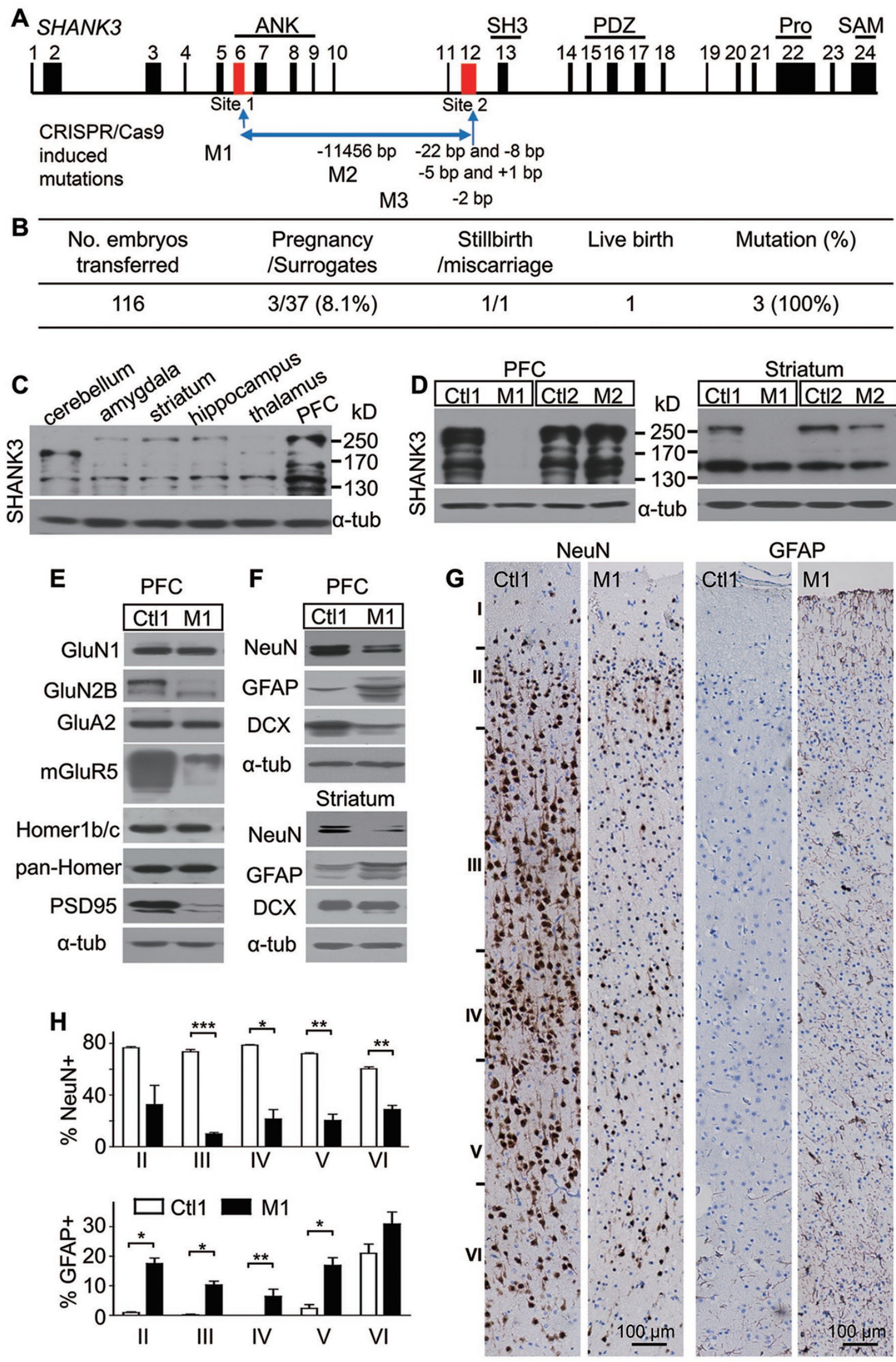
Figure 1 Generation and phenotypic analysis of SHANK3-mutant cynomolgus monkeys. (A) Schematic diagram of Macaca fascicularis SHANK3 gene, protein domains, Cas9/sgRNA targeting sites in exons 6 and 12, and confirmed mutations of SHANK3 in SHANK3 ${ }^{M 1-M 3}$ animals. The gene structure of monkey SHANK3 was deduced from the mRNA of XM_015456961 and a genomic contig of NC_022281 for SHANK3. (B) Summary of embryo transplantation and surrogacy in cynomolgus monkeys. (C) Brain region-specific expression profile of SHANK3 protein in wild-type P0 monkey. SHANK3 displays isoform-specific expression in different brain regions and is expressed at the highest level in PFC. (D) Western blot analysis of SHANK3 protein in the PFC and striatum of SHANK3-mutant monkeys. Ctl1 and Ctl2 are age-matched controls at gestational day 135 and full-term gestation, respectively. (E) Altered expressions of postsynaptic receptors and scaffold proteins in SHANK3 ${ }^{M 1}$ brain. Normal expressions of GluN1, GluA2, Homer1b/c, and pan-Homer but decreased expressions of GluN2B, mGluR5, and PSD95 were observed in the PFC of SHANK3 ${ }^{M 1}$. (F) Western blot analysis of NeuN, GFAP, and DCX in whole-cell lysates from PFC. SHANK ${ }^{M 1}$ showed decreased levels of NeuN and DCX but increased level of GFAP in the PFC. Western blot analysis was repeated at least three times independently. (G) NeuN and GFAP staining (brown) in layers I-VI of PFC. NeuN staining showed a strong signal in the nucleus and a weak signal in the cytosol. Nuclei were stained with hematoxylin (blue). There were fewer NeuN+ neurons and more GFAP+ astrocytes in the PFC of SHANK3 ${ }^{M 1}$. Scale bar, $100 \mu \mathrm{m}$. (H) Percentages of NeuN+ neurons and GFAP+ astrocytes in each layers of PFC. $n=6,{ }^{*} P<0.05$, ${ }^{* *} P<0.01$, and ${ }^{* * *} P<0.001$ by Student's $t$-test.

no apparent developmental defects in $S H A N K 3^{M I}$ and $S H A N K 3^{M 2}$ fetuses to explain their death. The brain and other somatic tissues of SHANK3 ${ }^{M 1}$ and $S H A N K 3^{M 2}$, and selected somatic tissues of $S H A N K 3^{M 3}$ were analyzed for targeted mutations at sites 1 and 2 . All three offsprings were positive for mutations at site 2 of exon $12(-22$ and -8 bp in $S H A N K 3^{M 1},-5$ and +1 bp in SHANK3 ${ }^{M 2}$, and -2 bp in $S H A N K 3^{M 3}$; Supplementary information, Figure S1C-S1F). A large deletion was detected in different tissues of $S H A N K 3^{M I}$ with a pair of primers spanning sites 1 and 2, as evidenced by a positive band around $500 \mathrm{bp}$ (Supplementary information, Figure S1C). Sequencing of this band confirmed an 11456 bp deletion between the two targeting sites (Supplementary information, Figure S1E). The small indels in different mutant animals were confirmed by deep amplicon sequencing (Supplementary information, Figure S1F). However, deep amplicon sequencing was unable to detect the large 11456 bp deletion. Using the semi-quantitative PCR analysis of genomic DNA, we estimated that $\sim 70 \%$ of the alleles carry the large deletion in $S H A N K 3^{M I}$, which is predicted to disrupt the expression of most SHANK3 isoforms based on the transcript pattern of SHANK3 in human and rodent $[6,10]$. Off-target analysis did not reveal any additional mutations (Supplementary information, Figure S1G).

The brain tissues from $S H A N K 3^{M 1}$ and $S H A N K 3^{M 2}$ provided a unique opportunity to investigate SHANK3 mutation-associated neuropathology. Shank3 displays a temporal- and spatial-specific expression for its isoforms in mouse brain with peak expression in striatum of postnatal day 14 (P14) brain (Supplementary information, Figure S2). In contrast, we found that SHANK3 showed the highest level of expression in the PFC of monkey at birth (P0), a developmental time point that corresponds to P14 in mice (Figure 1C). The distinct expression patterns of SHANK3 in mouse and monkey brains may reflect the evolutionary differences between the two species.
We performed western blot analysis using whole-cell lysates isolated from different brain regions of mutants and age-matched controls (Ctl1 for SHANK $3^{M I}$ and Ctl2 for $S H A N K 3^{M 2}$ ) using an antibody against the $\mathrm{C}$-terminus of mouse Shank3 that is predicted to detect most, if not all, SHANK3 isoforms of primates (Figure 1D) [11]. Compared to the control, no SHANK3 protein isoforms were detected in the cortex of $S H A N K 3^{M I}$ animal, indicating a complete loss of SHANK3 resulting from the $11 \mathrm{~kb}, 22$ and 8 bp deletions in the PFC (Figure 1D). In contrast, an isoform-specific deficiency of SHANK3 was revealed in the striatum of $S H A N K 3^{M 1}$ animal, in which the full-length isoform was absent but a short isoform was still present. In the $S H A N K 3^{M 2}$ brain, the full-length SHANK3 was decreased to a greater extent than the short isoform in the striatum. Interestingly, the majority of isoforms were not decreased but mildly increased in the cortex presumably by a compensatory mechanism. These findings are generally consistent with the mosaic nature of CRISPR/Cas9-mediated gene editing, which accounts for the various extents of disruption of the targeted gene in different brain regions. However, the isoform-specific expression of SHANK3 in different brain regions should also be considered.

Given the alteration of SHANK3-interacting proteins in Shank3-deficient mice [6, 7], we selected several synaptic proteins for western blot analysis in SHANK3-deficient monkey brain (Figure 1E, Supplementary information, Figure S3A and S3B). We found no apparent difference in the levels of GluN1, GluA2, Homer1b/c, and pan-Homer in the PFC between SHANK $3^{M 1}$ and control. However, a significant decrease was revealed for mGluR5 $(54.7 \% \pm 10.6 \%$ of ctl $1, n=5$, mean \pm SEM), GluN2B $(18 \% \pm 6.8 \%, n=3)$, and PSD95 $(15.5 \% \pm 2.6 \%$, $n=6)$ in the PFC of SHANK3 ${ }^{M 1}$ compared to the control. In striatal neurons of Shank3-deficient mice, Homerlb/c abnormally accumulates in the cytoplasm despite no significant increase in overall proteins [11]. Consistently, more 
cytoplasmic staining of Homer $1 \mathrm{~b} / \mathrm{c}$ was also observed in PFC neurons of SHANK3 $3^{M 1}$ animal compared with its control (Supplementary information, Figure S4A and S4B). We further co-stained SHANK3 and PSD95, and found apparent reductions in the staining intensity of the two proteins in the PFC of SHANK3 ${ }^{M I}$ (Supplementary information, Figure S5A), consistent with the western blot results (Figure 1D and 1E). The loss of SHANK3 and alterations in its interacting proteins in $S H A N K 3^{M I}$ brain verify the findings from other species $[11,12]$.

The abundant expression of SHANK3 in the control but a complete loss of SHANK3 in the PFC of the $S H A N K 3^{M I}$ animal led us to focus on the pathological outcome in this brain region. Immunohistochemical analysis revealed that the structure of cortical layers is comparable between $S H A N K 3^{M I}$ and its control. While the cell density of cortical layers did not differ between control and mutant PFCs, the cell bodies of $S H A N K 3^{M I}$ were smaller (Figure 1G). Strikingly, we found significantly fewer NeuN+ neurons in the PFC of SHANK $3^{M 1}(72.2 \%$ $\pm 1.2 \% \mathrm{NeuN}+$ cells/total cell counts for $\mathrm{Ctl} 1 \mathrm{and} 22.7 \%$ $\pm 5 \%$ for $S H A N K 3^{M I}$; Figure $1 \mathrm{G}$ and $1 \mathrm{H}$ ) with reduced soma size $\left(48.0 \pm 10.2 \mu \mathrm{m}^{2} \mathrm{NeuN}+\right.$ cell for $\mathrm{Ctll}$ and 22.7 $\pm 4.8 \mu \mathrm{m}^{2}$ for $\operatorname{SHANK3^{MI}}, n=6$ slices). On the contrary, there were significantly more GFAP+ astrocytes in the PFC of SHANK3 ${ }^{M I}$ animal compared to the control (Figure $1 \mathrm{G}$ and $1 \mathrm{H}$ ) based on quantitative analysis of $\mathrm{DAB}$ staining results. Double immunofluorescent staining confirmed fewer NeuN + cells in the PFC and the increased GFAP-labeling in the PFC and striatum of SHANK3 ${ }^{M I}$ brain (Supplementary information, Figure S4C and S4D). The histological studies of SHANK3 ${ }^{M 2}$ did not reveal any apparent abnormalities in the PFC (Supplementary information, Figure S5B), consistent with the finding of no apparent loss of SHANK3 in SHANK3 $3^{M 2}$ brain. The fewer NeuN+ neurons and more GFAP+ astrocytes in the PFC of $S H A N K 3^{M I}$ were further supported by western blot analysis (Figure 1F). Specifically, the NeuN protein was markedly reduced $(25 \% \pm 8.7 \%, n=5)$, whereas the GFAP protein was significantly increased $(357 \% \pm 144 \%$, $n=5$ ) in SHANK $3^{M I}$ PFC compared to control (Supplementary information, Figure S3C). Western blot analysis also showed a decreased level of Doublecortin (DCX; $65 \% \pm 3.7 \%, n=3$ ), a marker of progenitor neurons, in the PFC, but a normal level in the striatum of SHANK $3^{M I}$ brain (Figure $1 \mathrm{~F}$ and Supplementary information, Figure $\mathrm{S} 3 \mathrm{C}$ ). These results together suggest a brain region-specific neurogenesis defect in SHANK $3^{M I}$ mutants.

Given the altered synaptic receptors and PSD proteins in $S H A N K 3^{M l}$ brain, we used Golgi impregnation method to examine dendritic spines in the PFC of $S H A N K 3^{M l}$ and $S H A N K 3^{M 2}$ brains and observed a significant de- crease in dendritic spine density of the $S H A N K 3^{M I}$ but not $S H A N K 3^{M 2}$ mutants compared to control, whereas the length from the tip to the base of a spine was not significantly altered in both mutant animals (Supplementary information, Figure S6). These results are consistent with previous reports in Shank3-mutant mice or neurons derived from $S H A N K 3$-deficient patients documenting a reduced spine density $[11,12]$.

In summary, we successfully introduced various types of deleterious mutations in SHANK3 for the first time in cynomolgus monkeys by the CRISPR/Cas 9 genome editing method. SHANK3-deficient fetus showed a significant loss of NeuN+ neuronal cells accompanied with an increase of GFAP+ astrocytes, which has not been found in any line of Shank3-knockout mice, demonstrating a unique and critical role of SHANK3 in early brain development in primates. SHANK3 has been known as a scaffolding protein in synapses. However, our findings suggested an important role of SHANK3 in early developing brains in non-human primate. The mechanism by which SHANK3 regulates neurogenesis, in addition to the conventional synaptic function, in the prenatal brain remains to be elucidated. Our findings from SHANK3-deficient monkey predict that similar but milder changes may underlie the pathophysiology of human individuals with SHANK3 haploinsufficiency. The defects in early brain development and the reduced size of neuron soma are reminiscent of the neuronal defects in $\mathrm{MeCP} 2$ and PTEN ASD mouse models [1]. The pattern of molecular changes resulting from the SHANK3 deficiency is overall conserved between mouse and monkey brains, but the pathological changes are more prominent in SHANK3-deficient monkey. While these findings support the value of mouse models, they also underscore the importance of using non-human primates to model SHANK3 and other gene mutations causing ASD.

Materials and Methods are available in Supplementary information, Data S1, Tables S1 and S2.

\section{Acknowledgments}

We thank Drs Weixiang Guo, Xiaoming Wang and Zhiheng Xu for discussion, Ruxiao Xing, Xudong Liu and Jinquan Gao for technical assistance, Yafeng Luo (Guangzhou Yuanxi Biotech Co., Ltd) for animal resource and care, and Samuel Hulbert for critical reading of the manuscript. The project was supported by the Ministry of Science and Technology of China (2014CB942803 and 2016YFA0501000), the Strategic Priority Research Program of the Chinese Academy of Sciences (XDB02020400), and the National Natural Science Foundation of China (31110103907, 91332206 and 31490592).

Hui Zhao ${ }^{1,2, *}$, Zhuchi Tu ${ }^{1,3, *}$, Huijuan $\mathrm{Xu}^{1}$, Sen $\mathrm{Yan}^{3}$, Huanhuan Yan ${ }^{4}$, Yinghui Zheng ${ }^{1}$, Weili Yang ${ }^{3}$, 
Jiezhao Zheng ${ }^{5}$, Zhujun $\mathrm{Li}^{5}$, Rui Tian ${ }^{1}$, Youming $\mathrm{Lu}^{4}$, Xiangyu $\mathrm{Guo}^{3}$, Yong-hui Jiang ${ }^{6}$, Xiao-Jiang $\mathrm{Li}^{1,3,7}$, Yong Q Zhang ${ }^{1,2}$

${ }^{I}$ State Key Laboratory of Molecular Developmental Biology, Institute of Genetics and Developmental Biology, Chinese Academy of Sciences, Beijing 100101, China; ${ }^{2}$ University of Chinese Academy of Sciences, Beijing 100101, China; ${ }^{3}$ Guangdong-Hongkong-Macau Institute of CNS Regeneration, Ministry of Education CNS Regeneration Collaborative Joint Laboratory, Jinan University, Guangzhou, Guangdong 510632, China; ${ }^{4}$ The Institute for Brain Research, Collaborative Innovation Center for Brain Science, Huazhong University of Science and Technology, Wuhan, Hubei 430030, China; ${ }^{5} Y u a n x i$ Biotech Inc., Guangzhou, Guangdong 5010663, China; ${ }^{6}$ Department of Pediatrics and Department of Neurobiology, Duke University, Durham, NC 27710, USA; ' Department of Human Genetics, Emory University School of Medicine, Atlanta, GA 30322, USA

*These two authors contributed equally to this work.

Correspondence: Yong-hui Jiang ${ }^{\mathrm{a}}$, Xiao-Jiang Li ${ }^{\mathrm{b}}$, Yong Q Zhang ${ }^{\mathrm{c}}$

${ }^{a}$ E-mail: yong-hui.jiang@duke.edu

bE-mail: xli2@emory.edu

${ }^{\mathrm{c} E}$-mail: yqzhang@genetics.ac.cn

\section{References}

1 de la Torre-Ubieta L, Won H, Stein JL, et al. Nat Med 2016; 22:345361.

2 Amaral DG, Schumann CM, Nordahl CW. Trends Neurosci 2008; 31:137-145.

3 Watson KK, Platt ML. J Neurodev Disord 2012; 4:21.

4 Chen Y, Yu J, Niu Y, et al. Cell 2017; 169:945-955.

5 Liu Z, Li X, Zhang JT, et al. Nature 2016; 530:98-102.

6 Jiang YH, Ehlers MD. Neuron 2013; 78:8-27.

7 Monteiro P, Feng G. Nat Rev Neurosci 2017; 18:147-157.

8 Tu Z, Yang W, Yan S, et al. Sci Rep 2017; 7:42081.

9 Niu Y, Shen B, Cui Y, et al. Cell 2014; 156:836-843.

10 Zhu L, Wang X, Li XL, et al. Hum Mol Genet 2014; 23:1563-1578.

11 Wang X, Bey AL, Katz BM, et al. Nat Commun 2016; 7:11459.

12 Shcheglovitov A, Shcheglovitova O, Yazawa M, et al. Nature 2013; 503:267-271.

(Supplementary information is linked to the online version of the paper on the Cell Research website.) 